\title{
Patients with multiple myeloma referred for palliative care consultation: from retrospective analysis to future directions to improve clinical outcomes
}

\author{
Maria Caterina Pallotti ${ }^{1}$ (1) $\cdot$ Romina Rossi ${ }^{1}$ Emanuela Scarpi ${ }^{2} \cdot$ Monia Dall'Agata $^{2} \cdot$ Marianna Ricci $^{1}$. \\ Michela Ceccolini ${ }^{3} \cdot$ Sonia Ronconi ${ }^{3} \cdot$ Vanessa Valenti $^{1} \cdot$ Marco Maltoni $^{4,5} \cdot$ Giovanni Martinelli $^{6} \cdot$ Claudio Cerchione $^{3}$
}

Received: 31 March 2021 / Accepted: 8 September 2021 / Published online: 31 October 2021

(c) The Author(s) 2021

\begin{abstract}
Introduction New treatments have improved the overall survival of patients with multiple myeloma (MM). At diagnosis and during the course of the disease, patients often report pain and other symptoms. Given the long disease trajectory, psychologi$\mathrm{cal}$ and social issues are also frequent. Recently, the potential usefulness of early palliative care (EPC) was hypothesized in the area of hematology. We conducted a retrospective analysis of patients with MM referred to our institute for a palliative care (PC) consultation between January 2017 and June 2020. Our aim was to evaluate the main reasons (pain or other clinical symptoms) for the referral for a first PC consultation.

Methods We examined the main reasons for the first PC consultation, the number of PC consultations carried out, and the period of time between diagnosis, first and subsequent PC visits, and death. We also recorded information on the type of pain experienced and the treatments administered.

Results Of the 325 patients with MM followed at our hematology unit during the study period, 43 were referred for a PC consultation (39 for pain management and 4 to determine the most appropriate care setting (hospice or palliative homecare service)). Nineteen (44.2\%) of the 43 patients reported other symptoms in addition to pain. The median time between MM diagnosis and the first PC consultation was 473 days. Fifteen patients died, with a median 332 days between the first PC visit and death.

Conclusion Randomized studies on MM involving larger patient populations with access to EPC are needed to identify an effective clinical model to improve the management of patients with MM.
\end{abstract}

Keywords Multiple myeloma $\cdot$ Pain $\cdot$ Early palliative care $\cdot$ Symptoms $\cdot$ Hospice $\cdot$ Homecare

Maria Caterina Pallotti

mcaterina.pallotti@irst.emr.it

1 Palliative Care Unit, IRCCS Istituto Romagnolo Per Lo Studio Dei Tumori (IRST), Dino Amadori”, Via P. Maroncelli 40, 47014 Meldola, FC, Italy

2 Unit of Biostatistics and Clinical Trials, IRCCS Istituto Romagnolo Per Lo Studio Dei Tumori (IRST) "Dino Amadori", Meldola, FC, Italy

3 Hematology Unit, IRCCS Istituto Romagnolo Per Lo Studio Dei Tumori (IRST) "Dino Amadori”, Meldola, FC, Italy

4 Medical Oncology Unit, IRCCS Azienda Ospedaliero-Universitaria Bologna, Bologna, Italy

5 Department of Specialized, Experimental and Diagnostic Medicine, University of Bologna, Bologna, Italy

6 Scientific Directorate, IRCCS Istituto Romagnolo per lo Studio dei Tumori (IRST) "Dino Amadori”, Meldola, FC, Italy

\section{Introduction}

Multiple myeloma (MM) is the 14th most common type of cancer worldwide and represents $1.8 \%$ of all cancers in the USA. It is estimated that in 2020 , there were 32,270 new cases and 12,830 deaths from this disease. It is more frequent in males, and the median age at diagnosis for both sexes is 69 years [1].

MM is characterized by an increase in plasma cells, resulting in an excessive production of immunoglobulin proteins with different signs and symptoms due to ensuing multiple organ damage, e.g., anemia from abnormal bone marrow involvement, osteolytic bone lesions, hypercalcemia and other skeletal-related events (SREs) from bone damage, and renal failure from kidney problems [2, 3]. 
More than two-thirds of patients at diagnosis and nearly all during the course of the disease have myeloma bone damage and experience pain from osteolytic bone lesions, but different types of pain occur at different stages of the disease. In fact, if bone pain is more frequent at diagnosis and relapse, pain from chemotherapy-induced peripheral neuropathy develops during MM treatment (especially bortezomib, thalidomide, lenalidomide, and vinca alkaloids) and can worsen over time from the prolonged use of these drugs. Oropharyngeal mucositis and somatic pain caused by the use of growth factors are frequent during stem cell transplantation, after which post-herpetic neuralgia is often diagnosed. Furthermore, long survivors may experience late effects of treatments such as chronic pain, neuropathy, or asthenia, and may also develop psychological symptoms such as depression and anxiety [2-6].

Each type of pain requires careful evaluation of the symptom (i.e., cause, localization, type, intensity) to identify the most effective treatment. The management of MM bone pain follows a multidisciplinary approach involving not only the specialized PC team, but also an orthopedic specialist/physician and radiotherapist. Analgesic therapy consists of opioids associated with bisphosphonates, while radiotherapy or orthopedic surgery may be necessary in selected cases. Adjuvant drugs are also often needed [3, 6-8].

In recent years, although new drugs and treatment strategies have substantially improved the median survival (5-8 years) of patients with MMs, the disease remains incurable [9]. In its long trajectory, pain is a frequent occurrence, but there are many other issues to contend with such as cumulative toxicities from the multiple lines of therapy and the physical, psychological, and social aspects of the disease. Thus, the symptom burden is often high and negatively affects quality of life [10-15].

The current approach to the management of MM is to continue treatment for as long as the disease responds [15]. There is now evidence of the importance of establishing a timely and close collaboration between hematologists and PC specialists. Pain, fatigue, anorexia, nausea, depression, and anxiety are the most frequent symptoms of patients with hematologic malignancies. The burden of these symptoms, the long periods of hospitalization, the intensive treatments with significant toxicity, and the uncertain course of the disease are only some of the problems faced by patients. PC has been shown to be beneficial for individuals with solid tumors, and there is now evidence that it could also be useful for hematological malignancies $[16,17]$.

MM patients can experience years of side effects and symptoms, and within this context, early palliative care (EPC) could help to improve quality of life [15] by managing symptoms more effectively, reducing the need to discontinue specific treatments, and alleviating patient anxiety. It would provide much needed support for caregivers and could help to establish a relationship of trust between patients and PC clinicians, facilitating the development of advance care planning [15].

In Italy, PC services are available for inpatients and outpatients in hematology, oncology, and radiotherapy units in the form of a consultation with PC physician and nurse. For those in poorer clinical conditions or with a higher symptom burden, a PC homecare visit or hospice admission is proposed by a multidisciplinary team (physician, nurse, social worker, psychologist, and physiotherapist).

Within this context, we carried out a retrospective analysis at our cancer institute to evaluate the main reasons for the initial referral of MM inpatients and outpatients to our PC service. We consider our paper a starting point to encourage closer collaboration between hematologists and PC physicians in an effort to find the answers to the many open questions remaining in this area.

\section{Materials and methods}

We carried out a retrospective, single center study on patients with MM. All consecutive MM patients referred by a hematologist for a PC consultation between January 2017 and June 2020 were considered. Patients were identified through our institute's electronic medical records (CCE $\log 802.6$ of $\log 80$ S.r.l.) in which at least one access to the PC unit had been registered. Inclusion criteria were age $\geq 18$ years old, MM diagnosis, and $\geq 1 \mathrm{PC}$ consultation at our center.

We considered any setting, i.e., inpatient ward and outpatient clinic. Demographic data (sex, date of birth, date of death), date of MM diagnosis, and date of first consultation with PC team were retrieved. The stage of MM at the first consultation was noted, i.e., diagnosis/first-line treatment, second- or more-line treatment for relapse or progression, and follow-up. We analyzed the setting of the first PC consultation and the reasons behind the referral (presence of symptoms or patient management setting, i.e., hospice or palliative homecare service).

With regard to the clinical aspects, we reviewed the clinical notes in the electronic medical records and focused on the main reasons for the request of a first PC consultation. In particular, we recorded information on the type of pain (neuropathic, somatic, mixed) experienced and on the main cause of the pain (e.g., MM bone lesions, chemotherapyinduced neuropathy, herpes zoster-related neuralgia). We also evaluated the ongoing opioid and adjuvant therapy and any remodulation of the pain therapy needed at the first PC consultation. Other symptoms and needs reported by patients were also taken into consideration. Finally, we analyzed the number of $\mathrm{PC}$ consultations required by each patient, and the time lapse between subsequent visits, in particular, the time 
between the diagnosis and the first visit, the time between the first and other visits, and the time between the last visit and the death of the patient.

Given the descriptive nature of the study, a formal calculation of sample size and statistical power was not performed. Considering a preliminary analysis of clinical medical charts from previous years at our center, it was feasible to include about 40 patients. Descriptive statistical analyses were performed on the entire case series (absolute and relative frequency for categorical variables and means \pm standard deviation or median and quantiles for continuous variables). No interim analysis was planned. Institutional informed consent forms for the treatment of personal data had previously been signed by all patients at their first access to our center and included consent to use patient data, materials, and/or diagnostic test results for research purposes. The study was conducted in accordance with the principles laid down in the 1964 Declaration of Helsinki and was approved by the local independent Ethical Committee (Comitato Etico della Romagna, approval no. 8638/2020).

\section{Results}

Of the $325 \mathrm{MM}$ patients followed by our hematologists between January 2017 and June 2020, 43 (13.2\%) were referred a PC consultation. Twenty-three (53.3\%) were female, and the median age of the entire group was 71 years. Thirty-two (74.4\%) patients were evaluated at the outpatient PC clinic, and $11(25.6 \%)$ were seen as inpatients. At the first PC consultation, 20 (46.5\%) patients had just been diagnosed or were undergoing first-line treatment, 15 (34.9\%) had relapsed, were in progression, or were undergoing second- or more-line treatment, and $8(18.6 \%)$ were in followup. At the time of this analysis, $28(65.1 \%)$ patients were alive, and 15 (34.9\%) had died.

The main reason for referral to the PC clinic were pain management in 39 (90.7\%) patients and evaluation of the most appropriate PC setting (hospice or homecare PC) in $4(9.3 \%)$.

With regard to the $39 \mathrm{MM}$ patients evaluated for pain management, 17 (43.6\%) had somatic pain, 9 (23.1\%) neuropathic pain, and $13(33.3 \%)$ mixed pain. At the first PC visit, 24 (61.5\%) patients had pain from MM bone disease, $6(15.4 \%)$ had chemotherapy-induced neuropathic pain, one (2.6\%) had post-herpetic neuralgia, and 8 patients $(20.5 \%)$ reported pain for other reasons. Table 1 shows the main modifications needed to optimize the pain therapy and the type of opioid prescribed. Four patients were assessed at the PC clinic to identify the most appropriate care setting based on their clinical conditions and needs; 3 were referred for hospice admission, and one was assigned to the palliative homecare service. In the patients initially seen for pain
Table 1 Main modifications to the analgesic therapy and type of opioid prescribed during the first palliative care consultation

\begin{tabular}{ll}
\hline & No. patients (\%) \\
\hline Started paracetamol therapy alone & $2(5.1)$ \\
Started an opioid therapy & $5(12.8)$ \\
Opioid rotation & $10(25.7)$ \\
Increased opioid dose & $9(23.1)$ \\
Started adjuvant therapy alone & $6(15.4)$ \\
Scrambler therapy & $2(5.1)$ \\
Opioid rotation + added adjuvant therapy & $2(5.1)$ \\
No modified therapy & $3(7.7)$ \\
Opioid & \\
Tramadol & $4(10.2)$ \\
Tapentadol & $6(15.4)$ \\
Buprenorphine patch & $4(10.2)$ \\
Fentanyl patch & $7(18.0)$ \\
Oxycodone & $9(23.1)$ \\
Morphine sulfate & $1(2.6)$ \\
Fentanyl patch +oxycodone & $3(7.7)$ \\
Fentanyl patch + morphine sulfate & $1(2.6)$ \\
No opioid treatment around the clock & $4(10.2)$ \\
\hline
\end{tabular}

management, 5 subsequently required evaluation of the best PC setting. Three were referred for hospice care and 2 for palliative homecare. A total of 19 (44.2\%) patients experienced other symptoms in addition to pain; fatigue in $9(20.9 \%)$, constipation in $8(18.6 \%)$ and depression in 5 (11.6\%). Other symptoms often reported at the first PC visit were loss of appetite, cachexia, and insomnia.

The majority of patients had a median of 2 PC consultations (interquartile range $1-4$ ), while only 3 were seen more often by the PC team $(9,13$, and 28 PC visits). The median time between MM diagnosis and the first PC consultation was 473 days (interquartile range 57-1099). Details of the time between the first and subsequent PC visits, death, or last follow-up are reported in Table 2. In the 15 patients who died, there was a median of 332 days (interquartile range 60-480) between the first PC visit and death, while the median value (interquartile range 37-401) between the last visit and death was 224 days. The place of death was known for 11 patients; 6 died in a palliative care setting ( 5 in hospice and one at home followed by the palliative homecare service), 4 in an acute medical unit and one in the hematology ward of our institute.

\section{Discussion}

Pain is the most frequent symptom in patients with $\mathrm{MM}$ and is almost always present during the course of the disease [3]. The causes of this symptom differ, and the different types of 
Table 2 Time between diagnosis of multiple myeloma, first and subsequent PC visits, and follow-up or death

\begin{tabular}{llc}
\hline & No. patients & $\begin{array}{c}\text { Median value (days) } \\
\text { (interquartile range) }\end{array}$ \\
\hline Diagnosis and 1st PC consultation & 43 & $473(57-1099)$ \\
1st and 2nd PC consultation & 27 & $38(29-58)$ \\
2nd and 3rd PC consultation & 14 & $27(15-147)$ \\
3rd and 4th PC consultation & 10 & $20(14-29)$ \\
4th and 5th PC consultation & 5 & $14(14-20)$ \\
1st and 5th PC consultation & 5 & $112(73-117)$ \\
1st and last PC consultation & 27 & $73(38-224)$ \\
1st PC consultation and death & 15 & $332(60-480)$ \\
1st PC consultation and death/follow-up & 43 & $385(206-876)$ \\
Last PC consultation and death & 15 & $224(12-875)$ \\
\hline
\end{tabular}

$P C$, palliative care pain generally occur at different stages of the disease. Pain has a dramatic impact on patients, compromising mobility, independence, and quality of life [3, 8]. In agreement with literature data, the majority of the MM patients referred to our PC unit experienced pain, the main causes being MMrelated bone disease and chemotherapy-induced peripheral neuropathy. Many of the symptoms were present at diagnosis and at progression/relapse of the disease. As previously stated, the most frequent types of pain were somatic and mixed $[2-4,15]$. A large percentage of our study population required opioids, with or without paracetamol, and adjuvant therapy, for pain control.

Given that patients with MM often have severe pain or a difficult type of pain to treat, their management is best suited to a specialized PC team [3, 4, 8, 18]. Furthermore, the rapid development of MM treatment strategies has highlighted the need to carry out more in-depth research into biological issues such as the interactions between opioids and proteasome inhibitors or immunomodulating agents [3]. We believe that a closer collaboration between hematologists and PC specialists could help to answer the numerous open questions remaining in this area. In addition to pain, other symptoms and psychological and social needs often represent a burden for MM patients and their families, sometimes compromising the continuation of MM therapies and negatively affecting quality of life [10-13, 15].

In our study, 19 patients reported other symptoms in addition to pain at the first PC visit, and in around $25 \%$ of the population, the PC consultation was aimed at identifying the most appropriate care setting (hospice or palliative homecare service).

Seminal studies have shown that EPC improves symptom management and quality of life in patients with solid tumors [19-21]. Despite strong evidence of the need for PC in patients with hematologist malignancies, EPC is still widely underused in this setting. Consequently, patients tend to be referred for PC late in the course of their disease when they have numerous symptoms that negatively influence quality of life and are often still undergoing intensive treatment [22, 23].

Research has been performed into the potential usefulness of EPC in patients with hematologist malignancies, in particular, those undergoing hematopoietic stem cell transplantation [24-27]. El-Jawahri et al. conducted a randomized clinical trial on patients with acute myeloid leukemia receiving intensive chemotherapy and assigned to integrated palliative and hematology care twice a week during hospitalization or PC on demand. The results revealed an improvement in quality of life, psychological distress, and end-of-life care in patients undergoing the integrated care approach with respect to those receiving on-demand PC [28].

Although the importance of integrating hematology and $\mathrm{PC}$ is gaining ground, there is still some hesitancy because of a number of "barriers" such as the unpredictability of the course of the disease; the sudden transition of the disease from stable to progressive; and the sometimes unrealistic expectations of hematologists in relation to antitumor treatments. It is also difficult for the PC team to know when to give active support (e.g., antibiotics or transfusions) to patients who are not in an advanced stage of disease. There is still a limited understanding of PC interventions, a common misconception being that it is only for end-of-life care. Furthermore, the often close bond built up between hematologists and their patients means that it can be difficult to introduce another clinician (especially a PC specialist) into the care scenario [22, 23, 29, 30].

EPC has recently been proposed for use in patients with MM [14, 15]. In this setting, EPC could improve the standard of care during treatment, thus reducing the risk of discontinuation. Early intervention and better symptom management would also help to break down the psychological barriers of patients to PC. Although new models of integration between hematology and $\mathrm{PC}$ have been proposed considering different types of hematological diseases and 
different disease stages, it is clear that they must first be evaluated in studies involving large patient populations [14, $15,17,26]$.

In our study, there was great variability in the timing of patient referral to the PC unit and also in the number of the $\mathrm{PC}$ visits carried out for each patient. This was a result of the on-demand rather than routinely scheduled nature of the PC consultations. However, our results are nonetheless in line with those of the literature.

Porta-Sales et al. carried out a retrospective study analyzing, for the first time, the efficacy of EPC in patients with MM $(n=67)$. The median time lapse between MM diagnosis and first PC visit was 355 days, quite early considering that MM has a median survival of 7 years. The authors reported that the early involvement of the PC team led to a rapid reduction in the intensity of physical symptoms, an increase in the administration of opioids for pain, and a subsequent decrease in psychological aspects such as depression and anxiety [14]. They also concluded that an EPC approach would facilitate the decision-making process and, consequently, advance care planning [15, 23].

Our study suggests the usefulness of EPC to manage pain and symptoms in MM patients and to facilitate the decisionmaking process for the most appropriate end-of-life setting, thus reducing the need for intensive treatments or for access to emergency departments. Not proposing EPC to MM patients could lead to a higher rate of hospitalizations and anti-myeloma therapies in the last months of life, as reported in a retrospective review by Chalopin et al. [31].

The main limitations of our study include its retrospective design and the fact that the data were collected over a long period of time, with suboptimal completeness. Furthermore, the small sample size in a single center setting did not permit any potential associations to be identified. As our study was carried out during the initial period of activity of our PC unit, we evaluated pain and other symptoms exclusively on the basis of information collected in electronic medical records, without the systematic use of validated tools for the identification of the symptoms. Thus, we cannot rule out that some minor symptoms may not have been recorded. Our service has since implemented the use of validated tools for the clinical assessment (i.e., Edmonton Symptom Assessment System, Douleur Neuropathique 4, Memorial Delirium Assessment Scale).

Although a retrospective study methodology is not as strong as that of randomized trial, the findings from our retrospective analysis suggest that EPC could improve the management of MM patients. Further research is warranted in this setting. In conclusion, more randomized clinical trials involving larger populations and multiple centers, together with the standardization of access to EPC for patients with hematological malignancies, especially MM, are needed to improve patient quality of life.
Acknowledgements The authors thank Gráinne Tierney for editorial assistance.

Author contribution All of the authors conceived the idea for the paper and analyzed and interpreted the data. The first draft of the article was written by MCP, with revision for important intellectual content by RR and MM. All authors read and approved the final version of the manuscript for submission.

Data availability The datasets generated and/or analyzed during the current study are available from the corresponding author on reasonable request.

\section{Declarations}

Ethics approval and informed consent The study was carried out in accordance with the principles laid down in the 1964 Declaration of Helsinki. Ethics approval was given by the Comitato Etico della Romagna (Approval no. 8638/2020).

Consent to participate Informed consent was obtained from all patients participating in the study.

Consent for publication Informed consent was obtained from all patients.

Conflict of interest The authors declare no competing interests.

Open Access This article is licensed under a Creative Commons Attribution 4.0 International License, which permits use, sharing, adaptation, distribution and reproduction in any medium or format, as long as you give appropriate credit to the original author(s) and the source, provide a link to the Creative Commons licence, and indicate if changes were made. The images or other third party material in this article are included in the article's Creative Commons licence, unless indicated otherwise in a credit line to the material. If material is not included in the article's Creative Commons licence and your intended use is not permitted by statutory regulation or exceeds the permitted use, you will need to obtain permission directly from the copyright holder. To view a copy of this licence, visit http://creativecommons.org/licenses/by/4.0/.

\section{References}

1. US National Cancer Institute, Cancer stat facts: myeloma. Available at: https://seer.cancer.gov/statfacts/html/mulmy.html

2. Terpos E, Christoulas D (2018) Gavriatopoulou M (2018) Biology and treatment of myeloma related bone disease. Metabolism 80:80-90

3. Coluzzi F, Rolke R, Mercadante S (2019) Pain management in patients with multiple myeloma: an update. Cancers (Basel) 11(12):2037

4. Snowden JA, Ahmedzai SH, Ashcroft J, D’Sa S, Littlewood T, Low E, Lucraft H, Maclean R, Feyler S, Pratt G, Bird JM; Haemato-oncology task force of British Committee for standards in haematology and UK myeloma forum (2011) Guidelines for supportive care in multiple myeloma 2011. Br J Haematol 154(1):76-103.

5. Terpos E, Berenson J, Raje N, Roodman GD (2014) Management of bone disease in multiple myeloma. Expert Rev Hematol 7(1):113-125 
6. Rajkumar SV, Kumar S (2016) Multiple myeloma: diagnosis and treatment. Mayo Clin Proc 91(1):101-119

7. Michels TC, Petersen KE (2017) Multiple myeloma: diagnosis and treatment. Am Fam Physician 95(6):373-383

8. Davies MP, Fingas S, Chantry A (2019) Mechanisms and treatment of bone pain in multiple myeloma. Curr Opin Support Palliat Care 13(4):408-416

9. Rajkumar SV (2018) Multiple myeloma: 2018 update on diagnosis, risk-stratification, and management. Am J Hematol 3(8):981-1114

10. Boland EG, Boland JW, Ezaydi Y, Greenfield DM, Ahmedzai SH, Snowden JA (2014) Holistic needs assessment in advanced, intensively treated multiple myeloma patients. Support Care Cancer 22(10):2615-2620

11. Osborne TR, Ramsenthaler C, Schey SA, Siegert RJ, Edmonds PM, Higginson IJ (2015) Improving the assessment of quality of life in the clinical care of myeloma patients: the development and validation of the Myeloma Patient Outcome Scale (MyPOS). BMC Cancer 15:280

12. Ramsenthaler C, Kane P, Gao W, Siegert RJ, Edmonds PM, Schey SA, Higginson IJ (2016) Prevalence of symptoms in patients with multiple myeloma: a systematic review and meta-analysis. Eur J Haematol 97(5):416-429

13. Ramsenthaler C, Osborne TR, Gao W, Siegert RJ, Edmonds PM, Schey SA, Higginson IJ (2016) The impact of disease-related symptoms and palliative care concerns on health-related quality of life in multiple myeloma: a multi-centre study. BMC Cancer $16: 427$

14. Porta-Sales J, Guerrero-Torrelles M, Moreno-Alonso D, SarràEscarré J, Clapés-Puig V, Trelis-Navarro J, Sureda-Balarí A, Fernández De Sevilla-Ribosa A (2017) Is early palliative care feasible in patients with multiple myeloma? J Pain Symptom Manage 54(5):692-700

15. Samala RV, Valent J, Noche N, Lagman R (2019) Palliative care in patients with multiple myeloma. J Pain Symptom Manage 58(6):1113-1118

16. Vidal M, Hui D, Bruera E (2018) Palliative care in patients with leukemia: when and how? Curr Oncol Rep 20(12):95

17. El-Jawahri A, Nelson AM, Gray TF, Lee SJ, LeBlanc TW (2020) Palliative and end-of-life care for patients with hematologic malignancies. J Clin Oncol 38(9):944-953

18. Mercadante S (2019) The patient with difficult cancer pain. Cancers (Basel) 11(4):565

19. Temel JS, Greer JA, Muzikansky A, Gallagher ER, Admane S, Jackson VA, Dahlin CM, Blinderman CD, Jacobsen J, Pirl WF, Billings JA, Lynch TJ (2010) Early palliative care for patients with metastatic non-small-cell lung cancer. N Engl J Med 363(8):733-742

20. Parikh RB, Kirch RA, Smith TJ, Temel JS (2013) Early specialty palliative care-translating data in oncology into practice. N Engl J Med 369(24):2347-2351

21. Maltoni M, Scarpi E, Dall'Agata M, Zagonel V, Bertè R, Ferrari D, Broglia CM, Bortolussi R, Trentin L, Valgiusti M, Pini S, Farolfi A, Casadei Gardini A, Nanni O, Amadori D; Early
Palliative Care Italian Study Group (EPCISG) (2016) Systematic versus on-demand early palliative care: results from a multicentre, randomised clinical trial. Eur J Cancer 65:61-68.

22. Hui D, Bansal S, Park M, Reddy A, Cortes J, Fossella F, Bruera E (2015) Differences in attitudes and beliefs toward end-of-life care between hematologic and solid tumor oncology specialists. Ann Oncol 26(7):1440-1446

23. LeBlanc TW, Roeland EJ, El-Jawahri A (2017) Early palliative care for patients with hematologic malignancies: is it really so difficult to achieve? Curr Hematol Malig Rep 2(4):300-308

24. El-Jawahri A, LeBlanc T, VanDusen H, Traeger L, Greer JA, Pirl WF, Jackson VA, Telles J, Rhodes A, Spitzer TR, McAfee S, Chen YA, Lee SS, Temel JS (2016) Effect of inpatient palliative care on quality of life 2 weeks after hematopoietic stem cell transplantation: a randomized clinical trial. JAMA 316(20):2094-2103

25. Loggers ET, LeBlanc TW, El-Jawahri A, Fihn J, Bumpus M, David J, Horak P, Lee SJ (2016) Pretransplantation supportive and palliative care consultation for high-risk hematopoietic cell transplantation patients. Biol Blood Marrow Transplant 22(7):1299-1305

26. Ruiz M, Reynolds P, Marranzini R, Khan A, Ketterer J, Brahim A (2018) Role of early palliative care interventions in hematological malignancies and bone marrow transplant patients: barriers and potential solutions. Am J Hosp Palliat Care 35(11):1456-1460

27. El-Jawahri A, Traeger L, Greer JA, VanDusen H, Fishman SR, LeBlanc TW, Pirl WF, Jackson VA, Telles J, Rhodes A, Li Z, Spitzer TR, McAfee S, Chen YA, Temel JS (2017) Effect of inpatient palliative care during hematopoietic stem-cell transplant on psychological distress 6 months after transplant: results of a randomized clinical trial. J Clin Oncol 35(32):3714-3721

28. El-Jawahri A, LeBlanc TW, Kavanaugh A, Webb JA, Jackson VA, Campbell TC, O'Connor N, Luger SM, Gafford E, Gustin J, Bhatnagar B, Walker AR, Fathi AT, Brunner AM, Hobbs GS, Nicholson S, Davis D, Addis H, Vaughn D, Horick N, Greer JA, Temel JS (2021) Effectiveness of integrated palliative and oncology care for patients with acute myeloid leukemia: a randomized clinical trial. JAMA Oncol 7(2):238-245

29. Odejide OO, Salas Coronado DY, Watts CD, Wright AA, Abel GA (2014) End-of-life care for blood cancers: a series of focus groups with hematologic oncologists. J Oncol Pract 10(6):e396-e403

30. LeBlanc TW, El-Jawahri A (2015) When and why should patients with hematologic malignancies see a palliative care specialist? Hematology Am Soc Hematol Educ Program 2015:471-478

31. Chalopin T, Vallet N, Benboubker L, Ochmann M, Gyan E, Chaumier F (2020) Retrospective review of end-of-life care in the last month of life in older patients with multiple myeloma: what collaboration between haematologists and palliative care teams? BMJ Support Palliat Care mjspcare-2020-002293.

Publisher's note Springer Nature remains neutral with regard to jurisdictional claims in published maps and institutional affiliations. 